\title{
The applicability of Ki-67 marker for renal epithelioid angiomyolipoma: experience of ten cases from a single center
}

\author{
C. XU*, X. Z. JIANG ${ }^{*}$, , H. F. ZHAO, N. Z. ZHANG, L. MA, Z. S. XU \\ Department of Urology, Qilu Hospital of Shandong University, No.107, Wenhuaxi Road, Jinan, China \\ ${ }^{*}$ Correspondence: jiangxzh968@sdu.edu.cn \\ ${ }^{*}$ Contributed equally to this work.
}

Received September 8, 2012/ Accepted September 27, 2012

\begin{abstract}
In order to present our experience with 10 cases of renal epithelioid angiomyolipoma (EAML) and validate the applicability of Ki-67 (proliferation marker) for EAML, we reviewed medical records of 10 consecutive cases diagnosed EAML from January 2005 to February 2012 at our department. Clinical data were collected and analyzed and pathology slides were reviewed. The immunohistochemical reactions for Ki-67 were performed and tumors showed positive expression were estimated. Active follow-up was performed to investigate the association between $\mathrm{Ki}-67$ expression and the prognosis. The mean age and tumor size of the patients was 43.6 years (range 32-56) and $8.2 \mathrm{~cm}$ (range 2-15 cm), respectively. Seven were females while three were males. Radical nephrectomy was performed in 6 patients, partial nephrectomy in 3, and renal artery ligation in 1. The immunohistochemical reactions for HMB-45 (Human Melanoma Black), SMA (Smooth Muscle Actin) were positive but for S-100 were negative. The number of patients showing positive/negative Ki-67 expression was 5/5. The survival rate of the positive group was $20 \%(1 / 5)$ while $100 \%(5 / 5)$ of the negative group during the median follow-up time of 26.75 months (range 1-53). Recurrence, metastasis and death due to disease occurred in 1 (10\%), 3 (30\%) and 4 (40\%) patients, respectively. Higher expression (positive) of Ki-67 indicates the presence of EAML and poor prognosis of patients. Surgical excision including radical and partial nephrectomy is a considerable approach to the treatment for its malignant potential.
\end{abstract}

Key words: renal tumor, epithelioid angiomyolipoma, clinical characteristics, Ki-67 marker, prognosis

Renal angiomyolipoma (RAML) was first described by Fischer in 1911, and named by Morgan in 1951 [1]. On the basis of autopsy and population studies, the incidence rate of RAML has been estimated at 130 per 100,000 adults approximately [2]. RAML is a mesenchymal tumor of the kidney composed of blood vessels, muscle and fat. Classic renal angiomyolipoma (AML) follows a benign course in most cases. In contrast to the classic type, the epithelioid variant is mentioned with emphasis for its potentially aggressive behavior in the new edition of the 2004 WHO classification of renal tumors [3]. The epithelioid angiomyolipoma (EAML) comprises $<1 \%$ of all renal epithelial neoplasms [4] and about 7.7\% of renal angiomyolipoma [5]. As in most published studies about EAML were rare cases, only the clinical and pathological characteristics is still unclear.

Recently, Ooi and colleagues evaluated the use of Ki-67 in the pathological diagnosis of two cases of EAMLs demonstrating that both were strongly positive for Ki-67 while all four classic AML were completely negative [6]. However, during our clinical practice, we found that certain EAMLs would show variable nuclear staining of Ki-67 marker indicating even different prognosis of patients. Herein, we will present our experience with 10 cases with clinical and pathological characteristics of EAML and investigate the association between $\mathrm{Ki}-67$ expression and the prognosis.

\section{Patients and methods}

The medical records of patient presented with renal tumors from January 2005 to February 2012 were reviewed retrospectively. A total of ten patients underwent surgical treatment for the tumor and diagnosed as epithelioid angiomyolipoma at our department. Clinical data were collected and analyzed, including age, gender, clinical symptoms, tumor size and location, imaging study, treatment strategy.

The previous pathology slides with histopathological and immunohistochemistry reports were also reviewed. Representative formalin-fixed, paraffin-embedded blocks of tissue were obtained from all the 10 cases. Four-millimikron-thick 


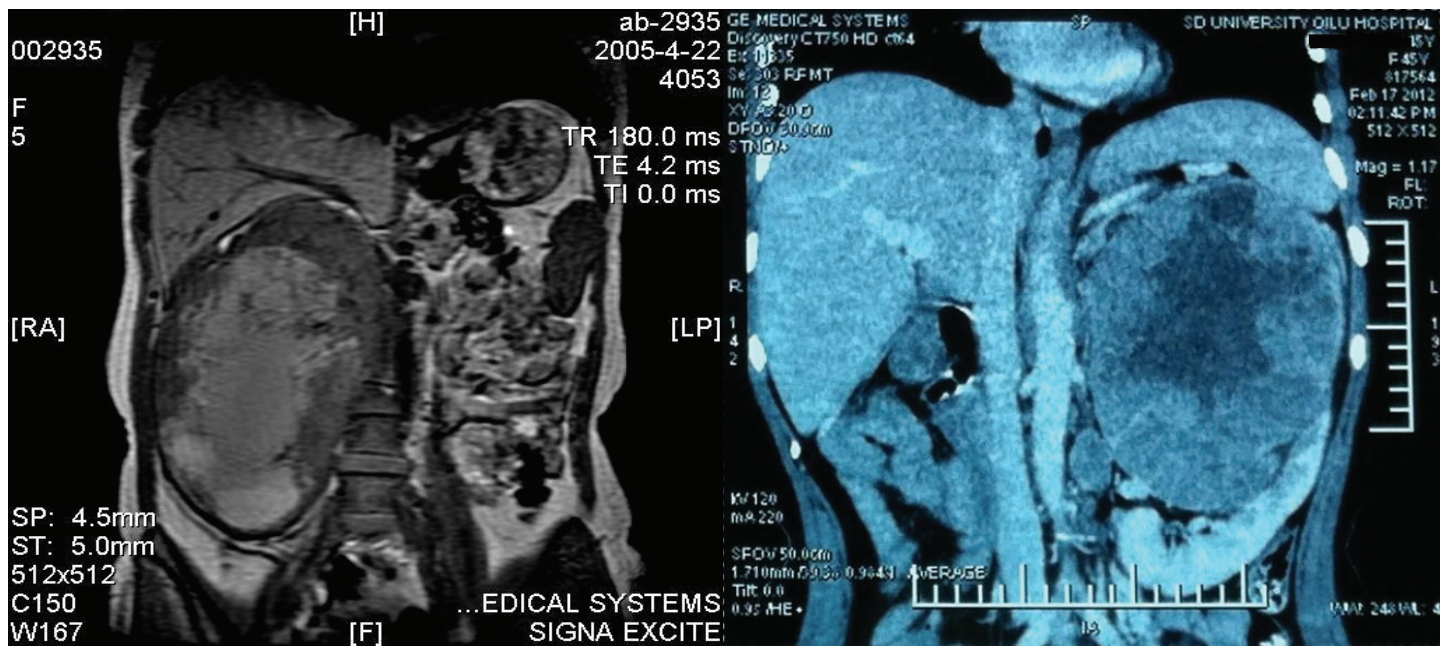

Figure 1. (Left) MRI scan revealing characteristic high signal intensity of fat on T1-weighted image. (Right) The enhanced CT scan showing a massive renal tumor with heterogeneous enhancement with different density of blood vessel, smooth muscle, and mature adipocytes.

sections were routinely stained with haematoxylin and eosin. The immunohistochemical reactions for HMB-45 (Human Melanoma Black), SMA (Smooth Muscle Actin), S-100 and Ki-67 were performed to detect EAML. More than $5 \%$ of cells showing staining were considered positive and the number of patients showing positive expression was estimated.

Active follow-up was performed in these patients. According to the survival status, patients were divided into two groups: living and dead. The association between the clinical and pathological characteristics and the prognosis was evaluated.

Analysis of data was performed with Statistic Package for Social Science (SPSS) 18.0 software. The comparisons of the general information such as age and tumor size between the two groups used Independent Sample T-test while gender and the tumor location were analyzed with Chi-square test. What's more, the role of Ki-67 in predicting the prognosis was assessed using Kaplan-Meier analysis and with Log Rank test. Any $\mathrm{P}<0.05$ was considered to indicate statistically significant differences.

Table 1. Comparisons of the general information between Living \& Dead group

\begin{tabular}{lccc}
\hline & $\begin{array}{c}\text { Living Patients } \\
(\mathrm{n}=6)\end{array}$ & $\begin{array}{c}\text { Dead Patients } \\
(\mathrm{n}=4)\end{array}$ & P-value \\
\hline Age & $39.67 \pm 8.87$ & $49.50 \pm 4.66$ & 0.079 \\
Tumor Size & $50.83 \pm 30.07$ & $128.75 \pm 29.55$ & 0.004 \\
Gender & & & 0.778 \\
$\quad$ Female & 4 & 3 & \\
$\quad$ Male & 2 & 1 & \\
Tumor location & & & 0.598 \\
$\quad$ Left & 4 & 2 & \\
$\quad$ Right & 2 & 2 & \\
\hline
\end{tabular}

\section{Results}

The mean age of the ten patients was 43.6 years (range 3256) while the mean tumor size was $8.2 \mathrm{~cm}$ (range $2-15 \mathrm{~cm}$ ), respectively. Seven were females while three were males (Female/Male Ratio was 2.3:1). The tumor was identified incidentally at a routine medical checkup in 3 patients; 5 presented with osphyalgia; 1 with fever and 1 with abdominal mass and constipation. Two patients underwent the complication of tuberous sclerosis complex (TSC) and both died within the follow-up time. Magnetic resonance imaging (MRI) could detect the characteristic high signal intensity of fat on $\mathrm{T} 1$-weighted image (Fig. 1). Computerized tomography (CT) before operation was performed in all ten patients. It could show a massive renal tumor with intermediate to low signal intensity and heterogeneous enhancement with different density of blood vessel, smooth muscle, and mature adipocytes, which was helpful for the diagnosis (Fig. 1).

Of the ten patients underwent surgical treatment for the tumor, in six cases was performed radical nephrectomy while in 3 with nephrectomy and renal artery ligation in 1 patient. All tumors were unilateral occurred in the left kidney in 6 patients while 4 in the right one. During the median follow-up time of 26.75 months (range 1-53, mean 25.90 months), recurrence and metastasis occurred in $10 \%(1 / 10)$ and $30 \%(3 / 10)$ of the patients, respectively. One female patient with local recurrence underwent radical nephrectomy as the first treatment and tumor resection for the recurrent tumor (Fig. 2). Unfortunately, she died 2 months later after she left our hospital. Moreover, one patient died of liver metastasis while 2 of lung (Fig. 2). Of the ten patients, all the four having recurrence or metastasis died and the other six survive free of disease until to date.

The comparisons of the general information between the surviving and dead groups are shown in Table 1. The 


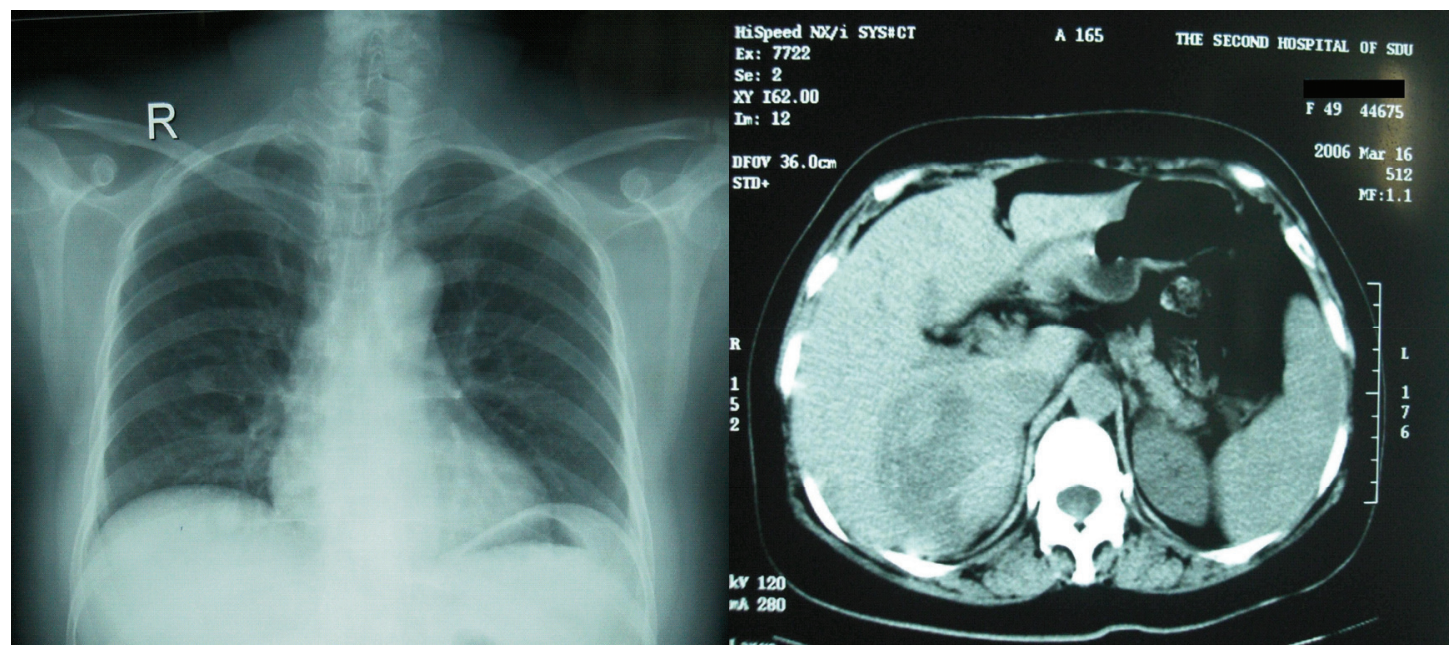

Figure 2. (Left) High-density masses could be found in the thoracic cavity with $\mathrm{X}$-ray chest film. (Right) The patient underwent local recurrence after 10 months of the radical nephrectomy.

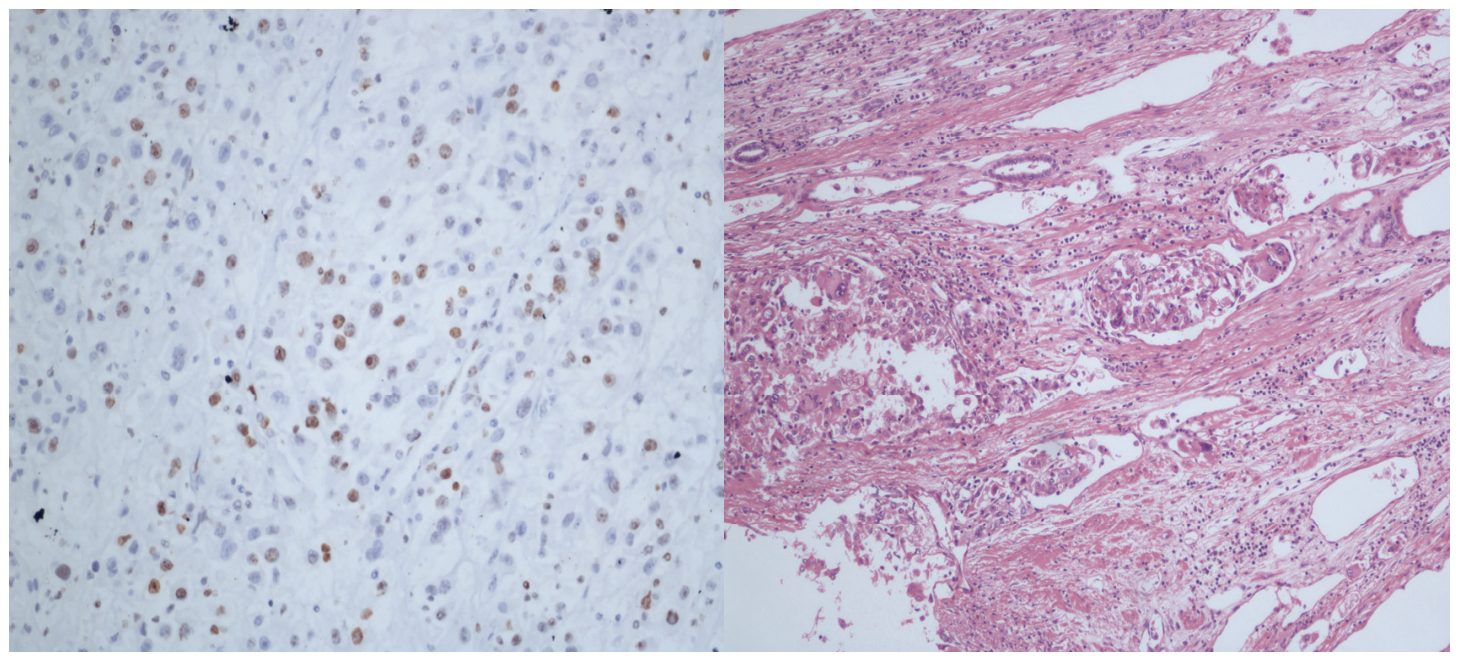

Figure 3. (Left) Positive Ki-67 expression with approximately 50\% of cells showing strong nuclear staining (magnification $\times 200)$. (Right) Tumor thrombosis could be discovered in the blood vessels. Haematoxylin-eosin stain (magnification $\times 100$ ).

mean tumor size (the greatest diameter) of the dead group $((128.75 \pm 29.55) \mathrm{mm})$ was larger than that of the surviving one $((50.83 \pm 30.07) \mathrm{mm}, \mathrm{P}=0.004)$. The mean age of the dead patients was approximately 10 years elder than that of the surviving ones. However, there were no statistically significant differences $(\mathrm{P}=0.079)$. Other clinical characteristics were uneventful with no statistically significant differences $(\mathrm{P}>0.05)$.

In all cases was performed microscopical examination. The tumors were predominantly composed of epithelioid cells, which were full of abundant eosinophilic cytoplasm. And, there were variable proportion of thick-walled blood vessels and adipocytes and scattered multinucleated giant cells. Nuclear pleomorphism and mitosis were seen and often hemorrhage and necrosis appeared. In certain cases, tumor thrombosis could be discovered in the blood vessels (Fig. 3). The immunohistochemical results showed that HMB- 45 and SMA were all positive yet S-100 was negative. However, the percentage of the cells showed strong nuclear staining with Ki-67 varied (range 1\%-50\%) (Fig. 3). The number of patients showing positive/negative Ki-67 expression was 5/5. The survival rate of the positive group was $20 \%$ (1/5) while $100 \%$ $(5 / 5)$ of the negative group. Kaplan-Meier analysis confirmed patients with positive Ki-67 expression would have worse prognosis than that with negative one with Log Rank test $(\mathrm{P}=0.016)$ (Fig. 4). 


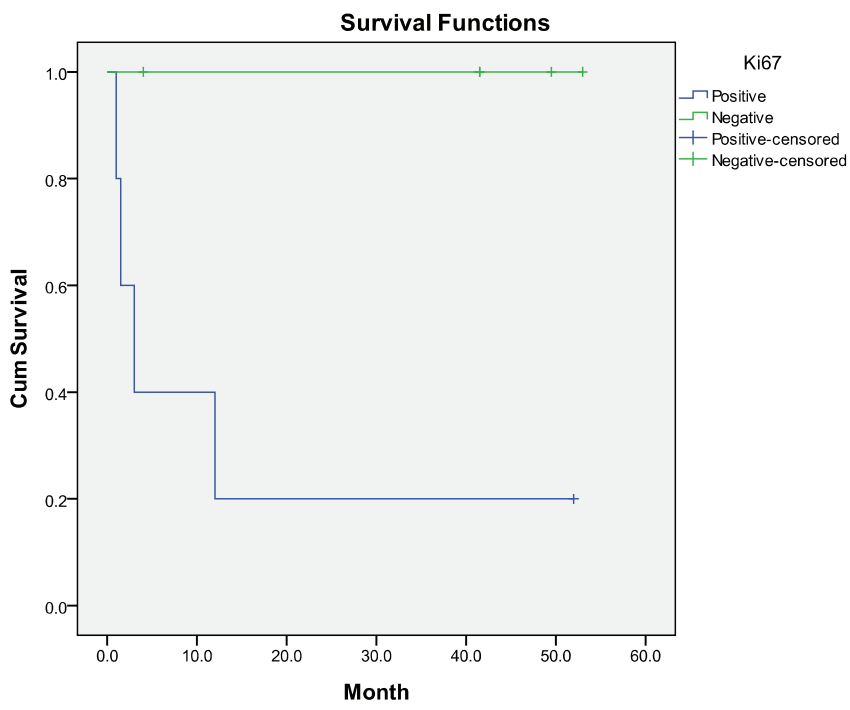

Figure 4. Kaplan-Meier analysis for the two groups of positive and negative Ki-67 expression.

\section{Discussion}

Renal AML occurred in two distinct groups of population. In patients without TSC, renal angiomyolipomas can be unilateral and are mostly in females from 40 to 70 years old. In the current study, the female/male ratio was 2.3:1 which indicated that women comprised the majority of this disease. On the other hand, angiomyolipomas are detected in approximately $40-80 \%$ of patients with TSC and are mostly bilateral and asymptomatic [7]. The age and gender of patients are uneventful. However, patients with TSC underwent malignant endpoint more frequently [8]. In our series, there were 2 patients (20\%) with TSC and both of them died of tumor metastasis.

The diagnosis of malignant EAML depends on radiographic imaging and histopathologic examination. Radiographic imaging alone is able to approach the initial diagnosis of AML, while the histopathologic examination helps to confirm the final diagnosis of EAML. The numerous fat-nonfat interfaces with ultrasonography produce an intensely echogenic lesion. Quantifying these echoes with computer assistance may aid in the discrimination of AML from other renal neoplasms. CT scan can detect fat densities ( -80 to -20 Hounsfield units) within the tumor, and the presence of fat is essentially pathognomonic for AML [9]. In our settings, CT scan before surgery could show a massive renal tumor with intermediate to low signal intensity and heterogeneous enhancement with different density of blood vessel, smooth muscle, and mature adipocytes, which was helpful for the diagnosis. The characteristic high signal intensity of fat on T1-weighted image of MRI scan will also assist in the diagnosis. However, as the presence of bleeding in any renal tumor will mimic the typical pattern of AML, MRI alone should not be considered for the final diagnosis of
AML. If fat could not be detected in the lesion using imaging studies, the tumor should be treated as malignant until a histopathologic diagnosis.

As for the histopathologic results of the microscopical examination with haematoxylin and eosin staining, malignant EAML is epithelioid smooth muscle cell in predominance with polygon shape, nucleus pleomorphism with atypical hyperplasia and sporadic mitosis. In certain cases, tumor thrombosis could be discovered in the blood vessels. With regard to the immunohistochemical examination, in renal angiomyolipoma, the epithelioid cells have preferential staining patterns for melanoma-associated proteins for HMB-50, NKI/C3, and tyrosinase in addition to HMB-45 [10]. Meanwhile, the epithelioid and adipocytic cells were CK, S100, and renin negative [10]. Therefore, HMB-45 and CD-68 positivity combined with cytokeratin negativity are of paramount importance for the correct diagnosis [11]. Positive Desmin and SMA expression will also help to lead to a convincing result $[12,13]$. These markers are very useful in discriminating between the AML and renal cell carcinoma, which is consistently negative for melanomaassociated markers and positive for epithelial markers [6]. In our series, the immunohistochemical results showed that HMB-45 and SMA were all positive yet S-100 was negative confirming the diagnosis of AML. However, these markers lack of convincing proof to diagnose the epithelioid sub-type. Therefore, it is necessary to find out an appropriate marker to discriminate epithelioid AML from classic type and to predict the prognosis accordingly.

The value of Ki-67 as a prognostic indicator has been established for breast cancer, soft tissue tumors, lung cancer, astrocytoma, and meningioma [6]. In 2004 and 2005, Chandrasoma and Quek demonstrated that higher Ki-67 expression occurred in the EAMLs, and Chandrasoma also suppose that it is a feature for the malignant types $[14,15]$. Ooi and colleagues evaluated the use of $\mathrm{Ki}-67$ in the pathological diagnosis of two EAMLs demonstrating that both cases of EAML were strongly positive for Ki-67 while all four classic AML were completely negative [6]. However, in Ooi's research, as only two patients were included, there might be a bias for the conclusion. What's more, during our clinical practice, we found that certain EAMLs would show variable nuclear staining when performing with Ki-67 marker. Whether this difference have influence on the prognosis of patients with EAML is still unclear. Therefore, in our series, we separated ten patients into two groups according to the expression status of Ki-67 to investigate the association between Ki-67 and patient's prognosis. The Kaplan-Meier analysis revealed the survival rate of the positive group was $20 \%(1 / 5)$ yet patients in the negative group were all alive (5/5) during the follow-up time. There was statistically significant difference between these two groups with Log Rank test $(\mathrm{P}=0.016)$.

The treatment strategy of EAML varied based on the individual patient. The size of the tumor may indicate the future course. Nese et al. suggested tumor size $>7 \mathrm{~cm}$ be 
a important prognostic parameter in predicting the disease progression [16]. In our series, patients of the living group had smaller tumor $((50.83 \pm 30.07) \mathrm{mm})$ than that of the dead ones $((128.75 \pm 29.55) \mathrm{mm})$. As for the tumors smaller than $10 \mathrm{~cm}$, the surgical processes including partial nephrectomy and or radical nephrectomy could be selected depending on the condition of patients. However, radical nephrectomy is recommended as the first choice when an EAML larger than $10 \mathrm{~cm}$ is detected. If patient's conditions permitted, adjuvant therapy is also welcomed. Once EAML have metastasis at diagnosis, systemic therapies including expectant treatment, selective arterial embolization, radical nephrectomy, partial nephrectomy, and follow-up regimen similar to that for renal carcinoma are considered. Whether targeted molecular therapy will show its outstanding impact on the EAML in prolonging the survival time, additional research on this issue is needed in the near future.

Generally, classic renal angiomyolipoma follows a benign course in most cases yet may have alarming properties: nuclear pleomorphism and mitotic activity, extension into the vena cava (IVC), and spread to regional lymph nodes, without malignant progression $[2,17]$. Mittal et al. reported a case of a benign renal AML with a tumor thrombus to the IVC, at 1 month after surgery, no thrombus within the IVC was detected with abdominal ultrasound and CT scan of the abdomen at 3 months post-operatively showed no evidence of recurrence [18]. However, patients who suffered from EAML would not be that fortunate. It presents a potentially malignant behavior in the clinical surveillance. A clinicopathologic study of 41 cases showed that recurrence and metastasis were seen in $17 \%$ and $49 \%$ of patients; $33 \%$ of patients died of disease [16]. Varma et al. reviewed 10 articles describing the clinical course of renal EAML found that almost all of the patients described, for whom there was a follow-up available, died of neoplastic progression of the disease [17]. The most common metastatic sites included the liver (63\%), lung (25\%), and mesentery (18.8\%) lymph node involvement happened in $24 \%$ of patients [16]. In our series, during the median follow-up time of 26.75 months, local recurrence developed in 1 patient and 2 patients were with lung metastasis while 1 in liver. All of them died of this disease in a few months. We considered that once recurrence or metastasis occurred, patients would follow a worse course with poor prognosis.

There were several strengths and limitations of this study. On one hand, we conducted a comprehensive study of several common clinical characteristics of this rare disease. On the other hand, we led an investigation and validation of the relationship of Ki-67 expression and patients' prognosis. However, as certain cases of this disease progress rapidly, we were unable to perform a comprehensive adjuvant therapy strategy after the surgery. Moreover, since the sample size with ten cases in analyzing the data is a pilot study, more clinicopathologic evidences should be confirmed in the future.

\section{References}

[1] MORGAN G S, STRAUMFJORD J V, HALL E J. Angiomyolipoma of the kidney. J Urol, 1951, 65: 525-527.

[2] EBLE J N. Angiomyolipoma of kidney. Semin Diagn Pathol, 1998, 15: 21-40.

[3] LOPEZ-BELTRAN A, SCARPELLI M, MONTIRONI R, KIRKALI Z. 2004 WHO classification of the renal tumors of the adults. Eur Urol, 2006, 49: 798-805. http://dx.doi. org/10.1016/j.eururo.2005.11.035

[4] FARAJI H, NGUYEN B N, MAI K T. Renal epithelioid angiomyolipoma: a study of six cases and a meta-analytic study. Development of criteria for screening the entity with prognostic significance. Histopathology, 2009, 55: 525-534. http://dx.doi.org/10.1111/j.1365-2559.2009.03420.x

[5] AYDIN H, MAGI-GALLUZZI C, LANE B R, SERCIA L, LOPEZ J I, et al. Renal angiomyolipoma: clinicopathologic study of 194 cases with emphasis on the epithelioid histology and tuberous sclerosis association. Am J Surg Pathol, 2009, 33: 289-297. http://dx.doi.org/10.1097/PAS.0b013e31817ed7a6

[6] OOI S M, VIVIAN J B, COHEN R J. The use of the Ki-67 marker in the pathological diagnosis of the epithelioid variant of renal angiomyolipoma. Int Urol Nephrol, 2009, 41: 559-565. http://dx.doi.org/10.1007/s11255-008-9473-1

[7] GUPTA C, MALANI A K, GUPTA V, SINGH J, AMMAR H. Metastatic retroperitoneal epithelioid angiomyolipoma. J Clin Pathol, 2007, 60: 428-431. http://dx.doi.org/10.1136/ jcp.2006.039503

[8] RADIN R, MA Y. Malignant epithelioid renal angiomyolipoma in a patient with tuberous sclerosis. J Comput Assist Tomogr, 2001, 25: 873-875. http://dx.doi.org/10.1097/00004728200111000-00008

[9] KIM J K, PARK S Y, SHON J H, CHO K S. Angiomyolipoma with minimal fat: differentiation from renal cell carcinoma at biphasic helical CT. Radiology, 2004, 230: 677-684. http:// dx.doi.org/10.1148/radiol.2303030003

[10] STONE C H, LEE M W, AMIN M B, YAZIJI H, GOWN A $\mathrm{M}$, et al. Renal angiomyolipoma: further immunophenotypic characterization of an expanding morphologic spectrum. Arch Pathol Lab Med, 2001, 125: 751-758.

[11] SVEC A, VELENSKA Z. Renal epithelioid angiomyolipoma-a close mimic of renal cell carcinoma. Report of a case and review of the literature. Pathol Res Pract, 2005, 200: 851-856. http://dx.doi.org/10.1016/j.prp.2004.09.004

[12] WEN J, LI H Z, JI Z G, MAO Q Z, SHI B B, et al. Renal epithelioid angiomyolipoma without obvious local progress in 10 years: a case report and literature review. Ir J Med Sci, 2011, 180: 557-560. http://dx.doi.org/10.1007/s11845-010$\underline{0616-\mathrm{X}}$

[13] INGLE A, KUMAR B, MENON S, BAKSHI G, DESAI S, et al. Epithelioid angiomyolipoma of kidney with atypical nuclear features and intranuclear inclusions on cytology. Diagn Cytopathol, 2011, 39: 278-282. http://dx.doi.org/10.1002/ dc. 21408

[14] CHANDRASOMA S, MOATAMED N, CHANG A, DANESHMAND S, MA Y. Angiomyolipoma of the kidney: expanding disease spectrum demonstrated by 3 cases. Appl Immuno- 
histochem Mol Morphol, 2004, 12: 277-283. http://dx.doi. org/10.1097/00129039-200409000-00016

[15] QUEK M L, SONI R A, HSU J, SKINNER D G. Renal epithelioid angiomyolipoma associated with hyperprolactinemia. Urology, 2005, 65: 797. http://dx.doi. org/10.1016/j.urology.2004.10.009

[16] NESE N, MARTIGNONI G, FLETCHER C D, GUPTA R, PAN, C. C, et al. Pure epithelioid PEComas (so-called epithelioid angiomyolipoma) of the kidney: A clinicopathologic study of 41 cases: detailed assessment of morphology and risk stratification. Am J Surg Pathol, 2011, 35: 161-176. http:// dx.doi.org/10.1097/PAS.0b013e318206f2a9

[17] VARMA S, GUPTA S, TALWAR J, FORTE F, DHAR M. Renal epithelioid angiomyolipoma: a malignant disease. J Nephrol, 2011, 24: 18-22. http://dx.doi.org/10.5301/JN.2010.5451

[18] MITTAL V, AULAKH B S, DAGA G. Benign renal angiomyolipoma with inferior vena cava thrombosis. Urology, 2011, 77: 1503-1506. http://dx.doi.org/10.1016/j.urology.2011.01.039 